\title{
The technique of dermoplasty to treat recurrent nasal polyps: preoperative evaluation, surgical details, postoperative endoscopic appearance and histopathologic analysis
}

\author{
George Anastasopoulos ${ }^{1}$, Dilyana Vicheva ${ }^{2}$, Eleni-Christina Tsiompanou ${ }^{3}$, Eleftherios Ferekidis ${ }^{4}$ \\ ${ }^{1}$ Department of Otolaryngology, "Euroclinic" Private Hospital, Athens, Greece \\ ${ }^{2}$ Department of Otolaryngology, Medical University, Plovdiv, Bulgaria \\ ${ }^{3}$ Department of Radiology, General Hospital of Rhodes, Rhodes, Greece \\ ${ }^{4}$ Department of Otolaryngology, National University of Athens, Eugenideion Hospital, Greece
}

\section{ABSTRACT}

BACKGROUND. The tendency of the nasal polyps for recurrence is well established and recognized. A potentially effective measure to reduce this tendency seems to be the replacement of the mucosa responsible for polyp growth.

MATERIAL AND METHODS. Since February 2012, we have used this method in fifteen patients with intractable polyposis. After a short course of preoperative medication, we performed a detailed computed scan analysis to study the anatomy of the ethmoidal complex and calculate the area and contour of the graft. Under general anaesthesia, we removed the mucosa of the nasal roof and replaced it with a split-thickness skin graft. For maintenance, only a short course of nasal drops every three months seems to suffice.

RESULTS. Excluding the first two cases due to a poor surgical technique, the rest of the cases may be considered successful. All patients experience a functional nose with restored nasal breathing and occasionally olfactory function with only a minimal pharmaceutical aid. Polyps still grow, but they show a much more benign course and are readily responsive to medical treatment. Interestingly, polyps present squamous metaplasia postoperatively. This feature needs further investigation.

CONCLUSION. The method of dermoplasty presents a high potential for control of polyp growth. With only an hour prolongation of surgical time, a minor increase in complication rate and a small increase in morbidity rate, the technique deserves further attention from the rhinological society.

KEYWORDS: nasal polyps, dermoplasty, endoscopic surgery, chronic rhinosinusitis

\section{INTRODUCTION}

It is well established and recognized that nasal polyps present a high rate of recurrence regardless of how radically they may be treated. Even if aggressive treatment is applied, such as radical extirpation of nasal tissues ${ }^{1}$ or topical application of antineoplastic agent ${ }^{2}$, the recurrence rate can reach up to $22.7 \%$ after 12 months ${ }^{3}$.

Certain subgroups of patients such as those suffering from bronchial asthma or Samter's triad exhibit an even more aggressive behaviour ${ }^{4}$. In these patients, multiple surgical operations are needed or protracted medical treatments usually with corticosteroids, which are not void of serious side effects ${ }^{5}$.

In this setting of uncertainty, the advent of the der- moplasty technique may provide a crude perhaps and non elegant solution, but effective nonetheless ${ }^{6}$. The removal of the part of the nasal mucosa responsible for the eruption of polyps renders this surgical technique radical enough, sparing at the same time most of the normal nasal tissues.

\section{MATERIAL AND METHODS}

Since January 2012, there have been fifteen recorded cases of application of the dermoplasty technique for the treatment of intractable nasal polyposis. Among the patients there are only four women. In the initial three cases, the technique was applied on both nostrils. In the last twelve cases, dermoplasty was applied unilaterally. 
All patients had suffered multiple surgical attempts at polyp removal and prolonged medical regimens due to frequent recurrences. Also, all patients were informed about the innovative nature of the technique and gave their consent to proceed with the operation.

Growing experience has led to the adaptation of a certain sequence of actions concerning preoperative preparation, surgical technique and postoperative management. Current practice began with the examination of the patient. Every effort was made to detect co-morbidities such as aspirin intolerance or bronchial asthma that lead to frequent polyp recurrences. The patient's polyps were staged and the patient was instructed to fill in a simple questionnaire regarding the severity of his/her symptoms. The patient was informed about the concept of the dermoplasty technique and, after obtaining his/her consent, the doctor instructed the patient to begin a nine-day regimen, consisting of oral administration of corticosteroids and antibiotics.

After completing the regimen, a high resolution computed tomography (CT) of the nose and paranasal sinuses was performed. The reason for waiting the completion of the regimen to perform the CT is because, at this stage, the anatomy of the operative field is clearer and it can be studied more easily. Furthermore, it is the time to estimate the surface and the contour of the skin graft needed.

The calculation of the area planned to be covered by the graft is made possible by studying the CT. First, the anteroposterior diameter is estimated studying the sagittal plane and calculating the distance between the posterior limit of the frontal ostium and the sphenoid sinus (including the roof of the sphenoid) (Figure 1).

The posterior rim of the frontal ostium is assigned the anterior limit of the working space as it is the anterior area most accessible by working with the $0^{\circ} \mathrm{en}-$ doscope. After estimating the anteroposterior diameter, we study the coronal plane taking measurements from at least ten points starting from the posterior rim of the frontal ostium and moving towards the sphenoid sinus (Figure 2). Using this data we can reconstruct and build a pattern for the skin graft.

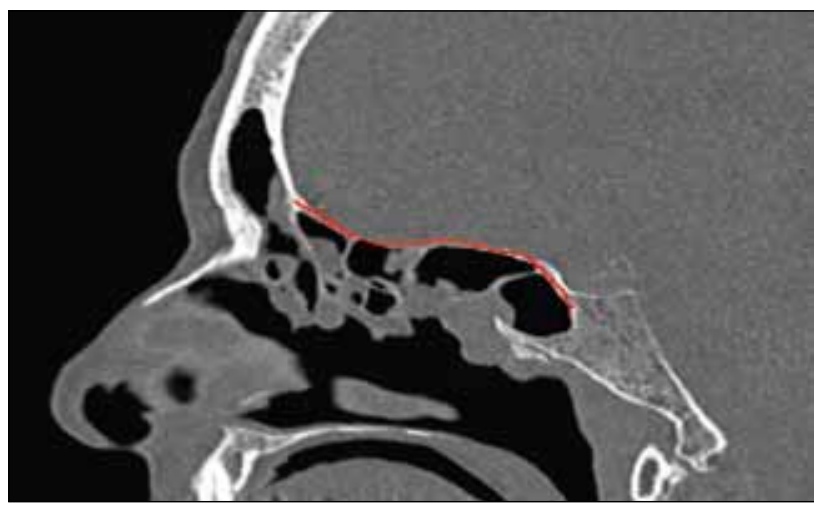

Figure 1 Calculating the anteroposterior diameter of the working area
After completing the pharmaceutical regimen, it is also the right time to decide which nostril will be chosen to apply the dermoplasty technique. Normally, unless other contraindications appear, we choose the side where we have free access to the axilla of the middle concha. The decision is taken after an endoscopic examination of the nose at the office.

The procedure lasts about four hours and it is performed under general anaesthesia. It involves complete ethmoidectomy on the dermoplasty side with removal of the bony ethmoidal septa and large part of the frontal wall of the sphenoid sinus. An effort is made to remove as much of the mucosa of the lamina papyracea, the fovea ethmoidalis and the lateral surface of the middle concha as possible, if present (Figure 3).

After completing radical ethmoidectomy and removal of the mucosa, we receive the split-thickness skin graft from the patient's thigh. The graft is trimmed to acquire the appropriate size and contour and, at the same time, multiple small incisions are made on its surface. These small cuts will prevent air and blood from accumulating between the graft and the bed and will also permit small mucosal remnants to gain access to the nasal cavity (Figure 4).

On the contralateral nostril, a typical functional endoscopic sinus surgery (FESS) is performed. No nasal packing of any kind is used. The patient stays overnight and leaves the hospital the next day. $\mathrm{He} / \mathrm{she}$ is instructed to take an antibiotic regimen for one week and to perform water saline rinses for the nose. Our current policy is to instruct our patients to use topical antibiotic/corticosteroid drops intranasally for one week every three months for maintenance.

\section{RESULTS}

Since January 2012, fifteen patients suffering from intractable polyposis have been operated using the dermoplasty technique. The first two cases were considered failures due to a poor operative technique. Also, in the first three cases, dermoplasty was applied

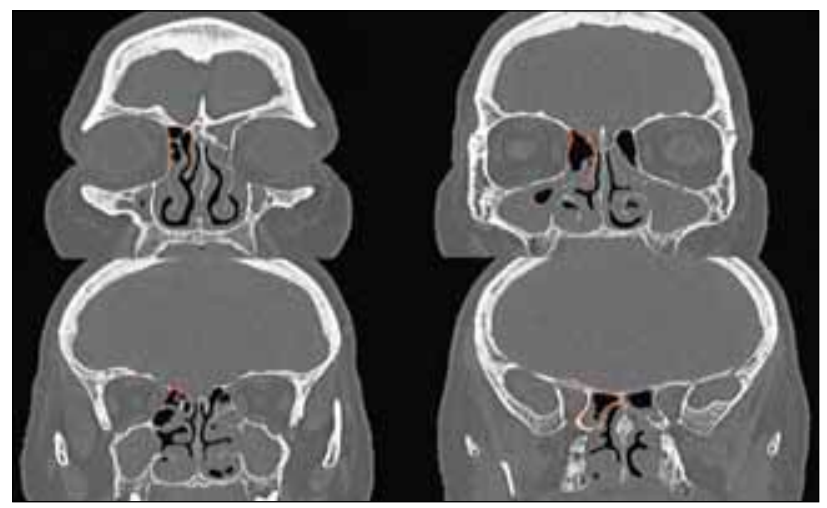

Figure 2 Multiple measurements at various points at the coronal plane 


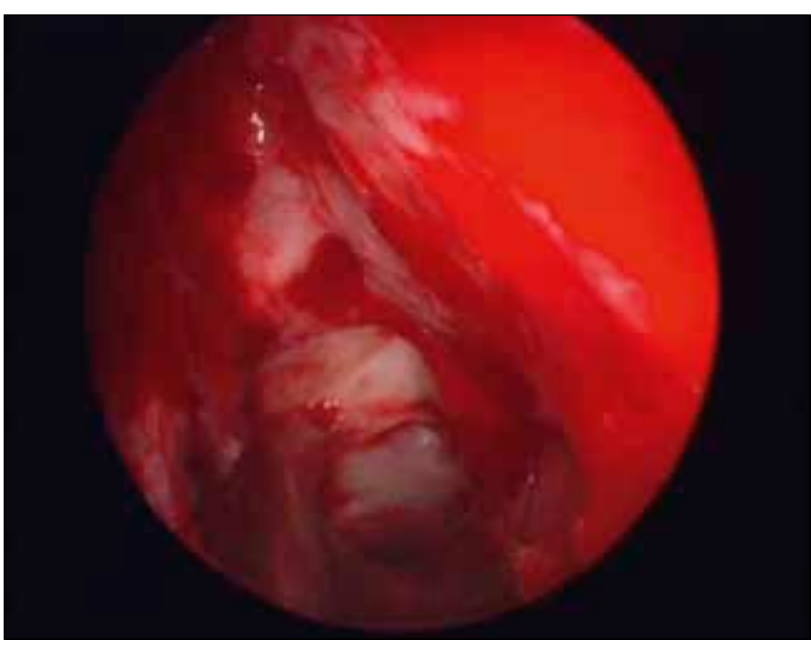

Figure 3 The operative field ready to receive the graft

on both nostrils. In the rest twelve cases dermoplasty was applied unilaterally.

Excluding the first two cases, the last thirteen cases may be studied as one group. All patients had an uneventful postoperative period with absence of serious complications and only minor disturbances from the wound in the leg.

In all cases the graft take was successful. The universal survival of the graft led to a minor late disturbance, which is the bad odor produced by the keratin debris of the skin graft starting usually after the first postoperative month. This minor complication, well known from other uses of skin grafts inside the nasal cavity, subsides in the following months (Figure 5).

All thirteen patients report being in a clearly improved situation compared to the same time interval after the previous surgical attempts. Also, all patients report a functioning nasal cavity with restored nasal breathing and only small periods of nasal congestion and all these with only minor pharmaceutical maintenance, consisting in topical use of antibiotic/corticosteroid drops for one week every three months.

Occasionally, certain patients experience even restoration to some degree of the olfactory function.

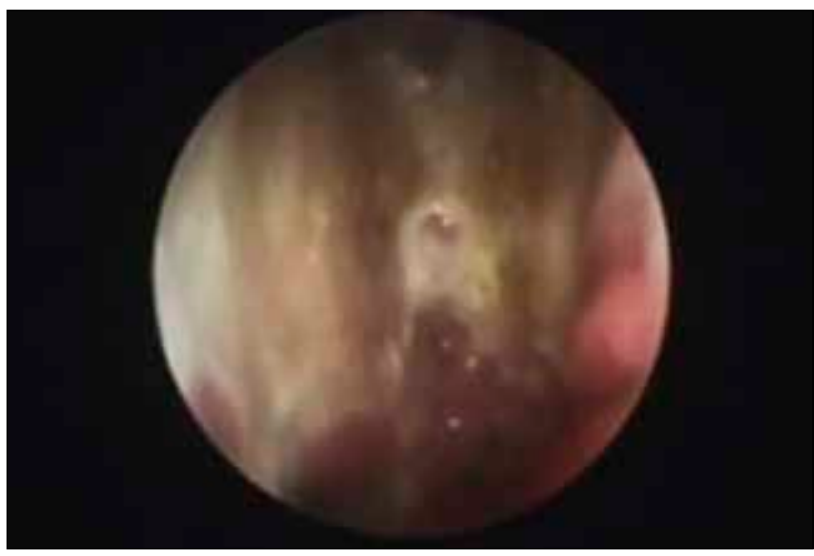

Figure $\mathbf{5}$ The skin graft several months after the insertion inside the nasal cavity

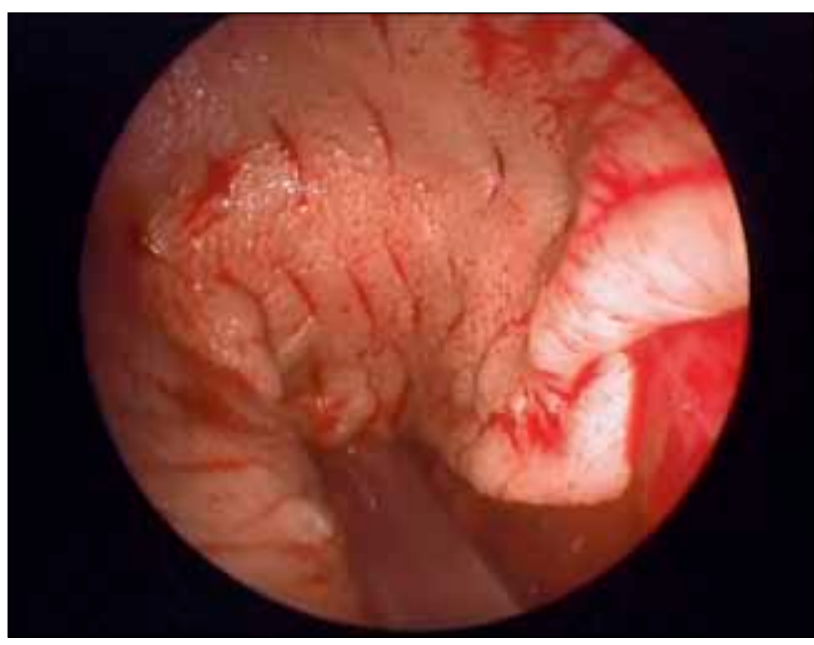

Figure 4 The skin graft in its final position

These bouts of the smelling function appear only in those patients who preoperatively experienced improvement of the olfactory function after receiving intense medication, usually oral corticosteroids.

All patients were under regular surveillance, approximately every three months for the first year, by means of nasal endoscopy in the office setting. Polyps never grow in the area covered by the skin graft, but still grow in other areas, nevertheless showing a much more benign course and a more readily response to topical medical therapy. Some biopsy specimens from the polyps growing postoperatively exhibit squamous metaplasia (Figure 6). This finding needs further investigation.

\section{DISCUSSIONS}

Until the advent of the endoscopic era in nasal surgery, little was added to the surgical treatment of nasal polyps since the time of Hippocrates. The founder of the medical science was using sponges and even a snare to remove polyps and hot iron to cauterize the remnants ${ }^{7}$.

The introduction of endoscopes in the armamentarium of Rhinology greatly improved both the diagnostics

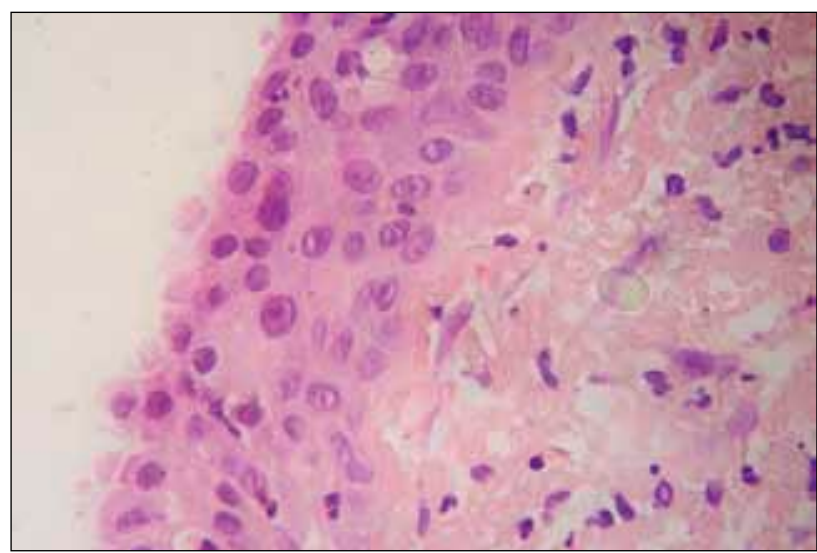

Figure 6 Nasal polyp with squamous metaplastic surface epithelium (haematoxylin and eosin stain, magnification 400x) 
and the operational abilities of rhinologists, establishing new expanded borders for the specialty. Nevertheless, referring to the problem of nasal polyposis, no significant improvement was recorded either when simple polypectomy was compared with functional endoscopic surgery (FESS) ${ }^{8}$ or when simple FESS was compared to radical or more extended applications of FESS ${ }^{9}$.

The idea of replacing the diseased mucosa with skin is not new. It is a well-established technique, both effective and safe, with many uses for the treatment of benign and malignant conditions. The combination of the concept of mucosa replacement with skin with the extended surgical abilities provided by the use of endoscopes gave birth to the technique of dermoplasty for recurrent polyposis.

Dermoplasty has two disadvantages when compared to simple FESS. First, the duration of the operation, which is at least one hour more compared to typical FESS. The situation was even worse during the first cases when we applied dermoplasty on both nostrils. Second, the wound in the leg that causes a delay for the patient to return to his/her previous activities.

Apart from these disadvantages, dermoplasty does not require any special equipment other than the electric dermatome, nor does it prolong the patient's hospitalization.

On the other hand, dermoplasty provides great benefits to the patients, keeping the airway paths open with minimal use of pharmaceutical support. No cases of atrophic rhinitis have been recorded among our patients or any other case of mucocele so far.

Several concepts are still under investigation. The unilateral application of dermoplasty was at first made necessary in order to reduce the duration of the operation. Our initial plan was to return after several months to apply dermoplasty on the second nostril. Until today, none of our patients has been in need for a second dermoplasty on the contralateral nostril.

Another concept under investigation is the histopathologic finding of squamous metaplasia in biopsy specimens of polyps appearing several months after the dermoplasty application, while the biopsy of the polyps of the specific patients during the operation had shown no such areas of metaplasia.

Several innovations may facilitate the technique of dermoplasty in the future, further reducing operational time. For example, the use of cultivated skin will save us from the wound in the leg and of course it will reduce operational time for about a quarter of an hour. Also, the introduction of robotic surgery in the field of rhinosurgery will add safety in such delicate operations.

The follow-up period may be short for the time being but, of course, here we speak about a new technique still under investigation with great potential for development if adopted by more colleagues with interest in Rhinology.

\section{CONCLUSIONS}

Dermoplasty for nasal polyps is a new technique showing promising results in the control of recalcitrant disease. The evolution of the technique and the adoption of further innovations require more experience to be gained through a larger number of cases, which in turn presupposes more colleagues with interest in Rhinology to test the efficacy of the method. Our effort is to popularize the technique and convince the scientific community of its efficacy.

\section{Conflicts of interests: None}

Contribution of authors: All authors have equally contributed to this work.

Ethical standards: Human studies have been performed in accordance with the ethical standards laid down in an appropriate version of the 1964 Declaration of Helsinki. All patients gave their informed consent for their inclusion in the study.

\section{REFERENCES}

1. Dessouky O., Hopkins C. - Surgical versus medical interventions in CRS and nasal polyps: comparative evidence between medical and surgical efficacy. Curr Allergy Asthma Rep., 2015;15(11):66. doi: 10.1007/s11882015-0566-5.

2. de Castro M.C., Assunção E., de Castro M.M., Araújo R.N., Guimarães R.E., Nunes F.B. - Effect of mitomycin C in eosinophilic nasal polyposis, in vivo: concentration of IL5 and GM-CSF, RT-PCR. Braz J Otorhinolaryngol,. 2006;72(1):38-42.

3. Bassiouni A., Wormald P.J. - Role of frontal sinus surgery in nasal polyp recurrence. Laryngoscope, 2013;123(1):36-41. doi: 10.1002/lary.23610. Epub 2012 Oct 15.

4. Rajguru R. - Nasal polyposis: current trends. Indian J Otolaryngol Head Neck Surg., 2014;66(Suppl 1):16-21. doi: 10.1007/s12070-011-0427-z. Epub 2011 Dec 29.

5. Leung R.M., Dinnie K., Smith T.L. - When do the risks of repeated courses of corticosteroids exceed the risks of surgery? Int Forum Allergy Rhinol., 2014;4(11):871-876. doi: 10.1002/alr.21377. Epub 2014 Aug 21.

6. Anastasopoulos G., Grigoriadis G., Papoutsi S. - Modified nasal dermoplasty technique for treatment of recurrent polyposis: preliminary results. J Laryngol Otol., 2013;127(6):595-598. doi:10.1017/ S0022215113000868. Epub 2013 Apr 26.

7. Colley P., Fried M.P., Tabaee A. - The History of Rhinology - From Ancient Times to the 21st Century. In: Sataloff R.T., Fried M.P., Tabaee A. - Sataloff's Comprehensive Textbook of Otolaryngology: Head \& Neck Surgery: Rhinology/Allergy and Immunology. JP Medical Ltd, 2015;p.313.

8. Devars du Mayne M., Prulière-Escabasse V., Zerah-Lancner F., Coste A., Papon J.F. - Polypectomy compared with ethmoidectomy in the treatment of nasal polyposis. Arch Otolaryngol Head Neck Surg., 2011;137(2):111-117. doi: 10.1001/archoto.2010.255.

9. Shen B., Liu L.T., Liu D., Guo Q.Y., Dong P. - Comparison of different surgical approaches of functional endoscopic sinus surgery on patients with chronic rhinosinusitis. Int J Clin Exp Med., 2014;7(6):1585-1591. eCollection 2014. 